\title{
The studying genetic regulation of the vaviloid type of spike branching in hybrid lines of hexaploid wheat
}

\author{
Adonina I.G. ${ }^{1 *}$, Zorina M.V. ${ }^{1,2}$, Shcherban A.B. ${ }^{1}$, Mehdiyeva S.P. ${ }^{3}$, Salina E.A. ${ }^{4}$ \\ ${ }^{1}$ Institute of Cytology and Genetics, SB RAS, Novosibirsk, Russia \\ ${ }^{2}$ Novosibirsk State University, Novosibirsk, Russia \\ ${ }^{3}$ Genetic Resources Institute of ANAS, Azerbaijan \\ ${ }^{4}$ Kurchatov Genomic Center of the Institute of Cytology and Genetics, SB RAS, Novosibirsk, Russia \\ *email: adonina@bionet.nsc.ru
}

Prolonged spikelet axes with additional flowers is observed in case of vaviloid type of spike branching in wheat. Forms with a vaviloid type of spike can occur as a result of distant hybridization. This trait is poorly researched. We studied lines obtained in Genetic Resources Institute of ANAS by crossing triticale with the hexaploid wheat Triticum aestivum L. var velutinum. Karyotyping was performed using GISH, FISH, and molecular-genetic analysis. For all plants with vaviloid type of spike the substitution of the wheat chromosome $2 \mathrm{D}$ by the $2 \mathrm{R}$ chromosome of rye or the telocentric chromosome $2 \mathrm{RL}$ is characteristic. A part of the plants contained 1-2 additional telocentric chromosomes of wheat 2DS. Only plants of line 857 had a hexaploid wheat karyotype $(2 n=6 x=42$, BBAADD) and a standard spike morphotype. According to our hypothesis, the gene/genes suppressing the manifestation of the vaviloid type of spike branching are localized on the long arm of chromosome 2D. As known from previous studies by other authors, some mutations in the gene $Q$ which is located on the long arm of chromosome 5A cause an elongation of the spikelet axis and thereby contribute to the formation of additional flowers on the spikelet axis. We found the absence of PCR amplification with primers matched to different regions of the $Q$-gene in the studied lines with vaviloid spike branching. These may be due to the deletion of the region of chromosome 5A carrying this gene.

Acknowledgments: This work was carried out as a part of the budget project No. 02592021-0012 and was funded by RFBR, project No. 20-016-00122. 